\title{
Erratum: One loop corrected conformally coupled scalar mode equations during inflation [Phys. Rev. D 96, 105003 (2017)]
}

Sibel Boran, Emre Onur Kahya, and Sohyun Park

(2) (Received 10 July 2018; published 19 July 2018)

DOI: 10.1103/PhysRevD.98.029903

Here we report a few numerical errors that we found after the publication of this manuscript. The conclusion of the paper is not affected by the correction of these numerical factors.

First, the $d_{i}$ 's in Eqs. (35-39) should be changed to

$$
\begin{gathered}
d_{1}=\frac{H^{D-4}}{(4 \pi)^{\frac{D}{2}}}\left\{-\frac{(D-2)}{2(D-4)(D-3)(D-1)}+\frac{1}{6} \gamma+\mathcal{O}(D-4)\right\}, \\
d_{2}=\frac{H^{D-4}}{(4 \pi)^{\frac{D}{2}}}\left\{\frac{8 D^{8}-127 D^{7}+782 D^{6}-2465 D^{5}+4386 D^{4}-4536 D^{3}+2848 D^{2}-1016 D+192}{2^{4}(D-4)(D-3)(D-1)^{3}}\right. \\
\left.-\frac{327}{80}-\frac{98}{9} \gamma+\mathcal{O}(D-4)\right\}, \\
\quad d_{3}=\frac{H^{D-4}}{(4 \pi)^{\frac{D}{2}}}\left\{\frac{45 D^{6}-650 D^{5}+3435 D^{4}-8520 D^{3}+10114 D^{2}-4456 D-178}{2^{5} 5(D-4)(D-3)(D-1)}\right. \\
\left.\quad-\frac{33421}{3600}+\frac{2163}{80} \gamma+\mathcal{O}(D-4)\right\}, \\
d_{4}=\frac{H^{D-4}}{(4 \pi)^{\frac{D}{2}}}\left\{-\frac{36 D^{7}-459 D^{6}+2070 D^{5}-7695 D^{4}+14290 D^{3}+316 D^{2}-20804 D+7440}{2^{3} 3^{2}(D-4)(D-3)(D-1)^{2}}\right. \\
\left.+\frac{2759}{540}-\frac{2327}{9} \gamma+\mathcal{O}(D-4)\right\}, \\
d_{5}=\frac{H^{D-4}}{(4 \pi)^{\frac{D}{2}}}\left\{-\frac{(D-2)^{2}}{(D-4)(D-3)(D-1)}+\frac{2}{3} \gamma+\mathcal{O}(D-4)\right\} .
\end{gathered}
$$

Second, some numerical factors in Eqs. (56) and (58) are affected by these changes in Eqs. (35-39) and should be changed to

$$
\begin{gathered}
\tilde{u}_{\text {nonlocal }}=\tilde{u}_{0}+\kappa^{2} \tilde{u}_{\text {1nonlocal }}+\mathcal{O}\left(\kappa^{4}\right), \\
\sim \frac{1}{\sqrt{2 k}}\left\{1-\frac{\kappa^{2} H^{2}}{\pi^{2}}\left[-\frac{3603}{2^{9} \cdot 15} a \ln (a)+\frac{50033}{2^{10} \cdot 15} a\right]\right\} . \\
u_{\mathrm{CC}} \sim \frac{1}{\sqrt{2 k}}\left\{\frac{1}{a}+G H^{2}\left[\frac{3603}{2^{5} \cdot 15 \pi} \ln (a)-\frac{50033}{2^{6} \cdot 15 \pi}-\left(\Delta c_{4}-\frac{3}{4}\right) \frac{\ln (a)}{a}\right]+\mathcal{O}\left(G^{2} H^{4}\right)\right\} .
\end{gathered}
$$

Third, the sentence in the beginning of Appendix A should be changed to "Table I uses $\tilde{\kappa}^{2} \equiv\left(\frac{D-2}{8(D-1)}\right)^{2} \kappa^{2}$ and Table III

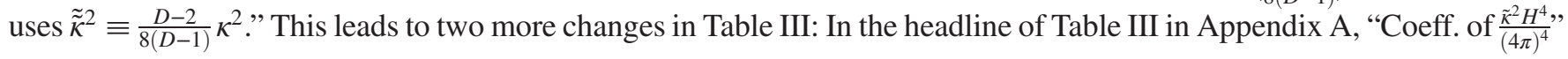


should be "Coeff. of $\frac{\tilde{\tilde{\kappa}}^{2} H^{4}}{(4 \pi)^{4}}$ " The second column of the first line of Table III should be 0 . The first entry of Table III now reads as follows:

\begin{tabular}{lc}
\hline External operators & Coeff. of $\frac{\tilde{\kappa}^{2} H^{4}}{(4 \pi)^{4}}$ \\
\hline$\left(a a^{\prime}\right)^{3} \square^{3} / H^{2}$ & 0 \\
\hline
\end{tabular}

Fourth, in the caption of Table VIII and Table X in Appendix D, “- $\frac{\tilde{u}(0, k)}{a^{2}} \frac{i H^{8}}{(4 \pi)^{2}}$ " should be changed to “- $\frac{\tilde{u}(0, k)}{a^{2}} \frac{i H^{8}}{(4 \pi)^{4}}$ ". That is, the power of $(4 \pi)$ is 4 not 2 .

Fifth, In Acknowledgements, we add: "S. B. and E. O. K. acknowledge the support from Istanbul Technical University, The Scientific Research Project (ITU-BAP) coordination unity with Project No. 39955." 